\title{
Inhibitor of PHA-Induced Lymphocyte Blastformation Generated from Maternal Lymphocytes during Early Pregnancy
}

\author{
Takuya Saito, Michiya Takada, Kunihiro Okamura \\ and NAKaO IsHIDA \\ Department of Bacteriology, Tohoku University School of \\ Medicine, Sendai
}

Sarto, T., Takada, M., OKamdRa, K. and Ishida, N. Inhibitor of PHAInduced Lymphocyte Blastformation Generated from Maternal Lymphocytes during Early Pregnancy. Tohoku J. exp. Med., 1975, 117 (1), 85-88 - The supernatant of phytohemagglutinin (PHA)-stimulated lymphocyte culture derived from pregnant women in the first two trimesters inhibited DNA synthesis of normal human lymphocytes after PHA induction. This soluble factor was heat-labile and the same factor could not be found in the supernatant generated from lymphocytes of pregnant women in 3rd trimester as well as from those of the non-pregnant. In contrast, the heatstable factor in the supernatant generated from lymphocytes of non-pregnant and pregnant women in all trimesters contained an stimulating factor on the DNA synthesis of normal human lymphocytes after PHA induction. An endogenous alternation in lymphocyte competence or an alternation in subpopulation of lymphocytes was suggested at the early stage of pregnancy. - immunosuppression; pregnancy; PHA-blastformation

The depression of cell-mediated immunity during pregnancy was partly explained by the presence of an inhibitor of the PHA-induced lymphocyte blastformation, contained in serum of the pregnant in 2nd and 3rd trimesters (Saito et al. 1975). Further studies on the diverse mechanisms by which an antigenetically foreign fetus is not rejected in the pregnant were conducted with soluble factors generated from lymphocytes as described by Maráz and Petri (1974). The present paper mainly concerns with the detection of an inhibitory factor which can be generated and released from lymphocytes in the culture fluid after PHA stimulation.

\section{Materials and Methods}

The generation of inhibitory or stimulatory factors from pregnant lymphocytes was conducted as follows. The lymphocytes were collected by gelatin sedimentation method from peripheral blood of the non-pregnant and the pregnant in Ist (16 weeks after gestation), 2nd (28 weeks) and 3rd (40 weeks) trimesters and adjusted to $1.0 \times 10^{6} \mathrm{cells} / \mathrm{ml}$ in RPMI 1640 medium containing $20 \%$ homologous human serum. Respective cultures in triplicate from one person containing $1.0 \mathrm{ml}$ medium received final $15 \mu \mathrm{g}$ of PHA.P (Difco) dissolved in $0.1 \mathrm{ml}$ medium and were incubated for the initial $48 \mathrm{hr}$ at $37^{\circ} \mathrm{C}$ in an atmosphere

Received for publication, May 14, 1975. 
of $5 \% \mathrm{CO}_{2}$. After $48 \mathrm{hr}$, these lymphocytes were lightly centrifuged and the medium was replaced by fresh RPMI 1640 medium with $20 \%$ human serum and incubated for further $24 \mathrm{hr}$ without PHA. The culture fluid (referred to as "supernatant" in the text) obtained after this $24 \mathrm{hr}$ incubation was examined for its immunosuppressive and immunostimulatory effects before and after heating at $56^{\circ} \mathrm{C}$ for $30 \mathrm{~min}$.

Immunosuppressive or immunostimulatory effect of the supernatant was determined by the effect of DNA synthesis in normal lymphocytes blastformation after PHA-P (Difco) activation, as was reported in a previous paper (Saito et al. 1975). For the assay, a culture of human lymphocytes $\left(1.0 \times 10^{8}\right)$ obtained from male blood donors in triplicate in $0.7 \mathrm{ml}$ of RPMI 1640 medium with $10 \%$ fetal calf serum received $0.3 \mathrm{ml}$ of undiluted pooled supernatant of triplicate cultures derived from one person. Respective cultures were stimulated by an addition of $15 \mu \mathrm{g}$ amount of PHA-P and during $66 \mathrm{hr}$ culture at $37^{\circ} \mathrm{C}$ in an atmo sphere of $5 \% \mathrm{CO}_{2},{ }^{3} \mathrm{H}$-thymidine incorporation into DNA was measured by pulse labelling for the last $18 \mathrm{hr}$. Details have already appeared in a previous paper (Saito et al. 1975).

\section{RESUlts}

Obtained results with heated supernatant are illustrated in Fig. 1. It is apparent in the figure that all heated supernatant contained a factor to stimulate the ${ }^{3} \mathrm{H}$-thymidine incorporation into DNA after the PHA induction, although the most significant stimulation was found with supernatants obtained from the nonpregnant and the pregnant in 3rd trimester. The same supernatant obtained from healthy male donors contained the same stimulatory factor (data not shown). Thus it seems apparent that peripheral lymphocytes or some subpopulation of such lymphocytes are endowed to synthesize and release such a stimulatory factor.

A more interesting finding, however, was the detection of a heat-labile inhibitory factor(s) from lymphocytes of the pregnant in 1st and 2 nd trimesters but not from 3rd trimester and the non-pregnant (Fig. 2). This result may suggest that

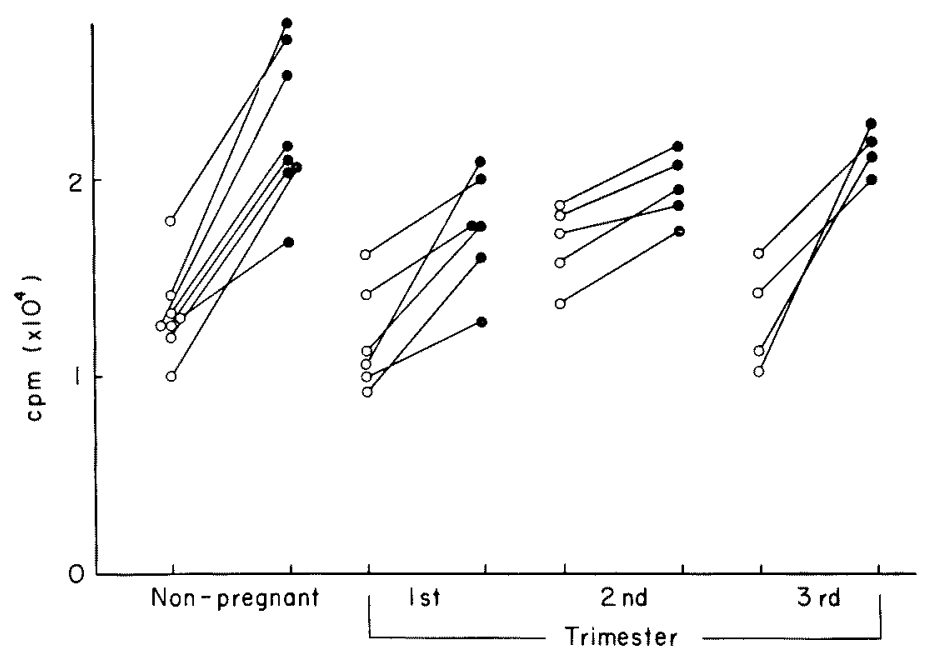

Fig. 1. Stimulatory effect of heated supernatant generated from lymphocytes of pregnant. and non-pregnant women on the PHA-induced blastformation.

- and 0 : PHA response with and without heated supernatant $(0.3 \mathrm{ml}$ in $1 \mathrm{ml}$ culture medium). 


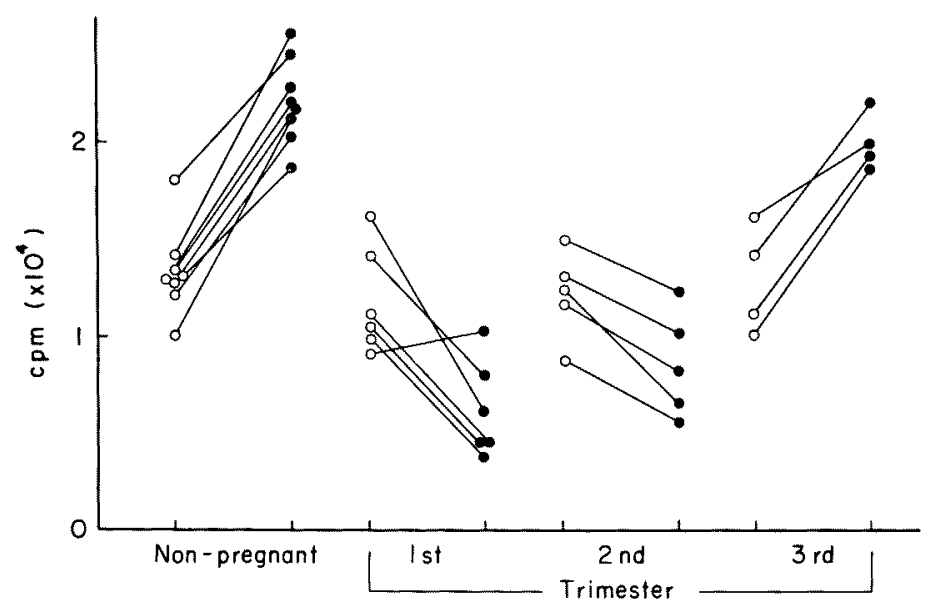

Fig. 2. Inhibitory effect of unheated supernatant generated from lymphocytes of pregnant women on the PHA-induced blastformation.

- and $\circ$ : PHA response with and without unheated supernatant.

unheated supernatant of the lymphocytes in 1st and 2nd trimesters should contain a fairly potent inhibitor, when the fact that the same supernatant is containing heat-stable stimulatory factor (Fig. 1) is taken into consideration. In Fig. 2, the unheated supernatants obtained from the non-pregnant and the pregnant in 3rd trimester were found to be stimulative, exactly concordant with the results shown in Fig. 1. Either of them did not contain a detectable amount of inhibitory factor.

\section{Discussion}

The presence of immunosuppressive factor in the sera of 2nd and 3rd trimesters was established in our previous paper (Saito et al. 1975). Although the factor contained in the 2nd trimester seemed to be different in the mechanism of action from that in the 3 rd trimester, both of them were found to be heat stable. Another kind of immunosuppressive factor was found in the supernatant of lymphocyte culture as described in this report. This factor was heat labile and can be generated from lymphocytes of the pregnant in 1st and 2nd trimesters but not in 3rd trimester. Thus the serum inhibitory factor and lymphocyte-originated inhibitory factor must be different each other when heat stability and the detection period during pregnancy is taken into account. The presence of the latter factor was initially described by Maráz and Petri (1974) and was designated as to "lymphocyte DNA synthesis inhibitor (DSI)" However, they did not collect serum specimens from the pregnant in a systematic manner as has been conducted in this study. A new finding in this study is that when unheated lymphocyte supernatants were examined for their inhibitory effect, the most remarkable inhibitory effect was demonstrated with 5 out of 6 lymphocyte superntants of lst trimester and almost the same but low grade inhibitory effect 
was found with 5 out of 5 specimens of 2 nd trimester but was not detectable with 4 out of 4 specimens of 3rd trimester (Fig. 2). Such an endogenous alternation in lymphocyte competence or an alternation in subpopulation of lymphocytes during pregnancy can be conceivable, but the real mechanism to explain such an alternation during early pregnancy in contrast to an alternation in the level of serum inhibitory factor during late pregnancy is still unknown. Whether these two phenomena are coupled or not and whether these two are mainly involved in the deprssion of cell-mediated immunity during pregnancy are the matter of concern at present and chemical characterization of these factors is now under study.

\section{Acknowledgments}

This work was supported in part by grant from the Ministry of Education of Japan.

\section{References}

1) Maráz, A. \& Petri, I.B. (1974) Intrinsic alternation of lymphocyte reactivity in women with normal pregnancy or tumor of placental origin. Cell. Immunol., 10, 496499.

2) Saito, T., Takada, M. \& Ishida, N. (1975) Inhibition of PHA-induced lymphocyte stimulation by factors in maternal serum during late pregnancy. Tohoku $J$. exp. Med., 116, 77-80. 